Volume 360, Number 1, January 2008, Pages 331-346

S 0002-9947(07)04266-3

Article electronically published on May 16, 2007

\title{
HOLOMORPHIC GERMS \\ AND THE PROBLEM OF SMOOTH CONJUGACY IN A PUNCTURED NEIGHBORHOOD OF THE ORIGIN
}

\author{
ADRIAN JENKINS
}

\begin{abstract}
We consider germs of conformal mappings tangent to the identity at the origin in $\mathbf{C}$. We construct a germ of a homeomorphism which is a $C^{\infty}$ diffeomorphism except at the origin conjugating these holomorphic germs with the time-one map of the vector field $V(z)=z^{m} \frac{\partial}{\partial z}$. We then show that, in the case $m=2$, for a germ of a homeomorphism which is real-analytic in a punctured neighborhood of the origin, with real-analytic inverse, conjugating these germs with the time-one map of the vector field exists if and only if a germ of a biholomorphism exists.
\end{abstract}

\section{INTRODUCTION}

We consider germs of conformal mappings at the origin which are tangent to the identity. Such germs are represented by power series of the form

$$
F(z)=z+a_{m} z^{m}+O\left(z^{m+1}\right),
$$

where $a_{m} \neq 0$. We say that two germs $f$ and $\tilde{f}$ of the form (1.1) are equivalent (or conjugate) to one another if we can find a mapping $H$ so that

$$
\left(H^{-1} \circ f \circ H\right)(z)=\tilde{f}(z) .
$$

We can talk about formal, topological, smooth, or holomorphic equivalence depending on the map $H$. The thrust of this paper is as follows: given any two maps $f$ and $\tilde{f}$ of the form (1.1), we can find a local homeomorphism $H$ which is $C^{\infty}$ off the origin conjugating $f$ with $\tilde{f}$. However, in the case that $m=2$ and $\tilde{f}(z)=\frac{z}{1-z}$, real-analytic equivalence in a punctured neighborhood of the origin will imply holomorphic equivalence in a full neighborhood of the origin. Precise statements will follow, but first, we look at some past results.

It is known that all such mappings (1.1) are topologically equivalent to the mapping

$$
F_{0}=g_{V}^{1},
$$

the time-one map of the vector field $V(z)=z^{m} \frac{\partial}{\partial z}$; this was proven by Shcherbakov (see [5]). Moreover, Shcherbakov shows that the conjugating mapping $h$ taking $f$ to $g_{V}^{1}$ can be written in the form $h(z)=z+\hat{h}(z)$, where $\hat{h}$ satisfies certain additional estimates (which are stated in our own theorem, below). Returning to the mapping

Received by the editors May 18, 2005 and, in revised form, February 7, 2006.

2000 Mathematics Subject Classification. Primary 30D05.

Key words and phrases. Smooth conjugacy, holomorphic germ, time-one map.

(C)2007 American Mathematical Society Reverts to public domain 28 years from publication 
$f$ itself, in the case that $m=2$, it is trivial to show that a necessary condition for formal (and hence holomorphic) equivalence of (1.1) with (1.3) is that

$$
\frac{a_{3}}{a_{2}^{2}}=1 \text {. }
$$

Thus, the continuous and holomorphic classifications for these germs do not agree. In fact, as Voronin shows in [6], the holomorphic classification admits functional moduli (the so-called Ecalle-Voronin moduli), and as a consequence, those germs (1.1) which are holomorphically equivalent to (1.3) are quite rare indeed. However, on sector regions of the form

$$
S_{j}=\left\{z:|z|<r,\left|\arg z-\frac{\pi j}{m-1}\right|<\frac{\pi}{2(m-1)}+\alpha\right\}, \quad j=1, \cdots, 2(m-1),
$$

where $r>0$ is sufficiently small and $0<\alpha<\frac{\pi}{2(m-1)}$, the classifications do agree (see [2]).

Later, Martinet and Ramis (in [3]) showed that even a $C^{1}$ equivalence between (1.1) and (1.3) will imply either a holomorphic or antiholomorphic equivalence (although the statement and proof which they give is incomplete, it can be corrected easily). In the case that $f(z)=z+z^{2}$, Ahern and Rosay [1] have proven that any germ of an entire function which is $C^{6}$ conjugate to $f$ must in fact be holomorphically equivalent to $f$. However, there remains some space between the result of Shcherbakov and that of Martinet and Ramis. Furthermore, as Ahern and Rosay mention in [1, the Ecalle-Voronin moduli are so difficult to compute as to be almost useless for precise examples.

The goal of this paper is two-fold. First, we shall generalize the theorem by Shcherbakov, mentioned above, as follows.

Theorem 1.1. Suppose that $f$ is a germ of a holomorphic map at $0 \in \mathbf{C}$ of the form (1.1), and suppose that $F_{0}$ is the time-one map of the vector field $v(z)=z^{m} \frac{\partial}{\partial z}$. Let $\varepsilon>0$ and $\delta>0$ be given. Then, there exists a homeomorphism

$$
H=p+h,
$$

defined in a small neighborhood of the origin, where $p$ is a holomorphic polynomial of degree at most $m-1$, and furthermore where $H$ is a $C^{\infty}$ diffeomorphism except at the origin, satisfying the following:

$$
H \circ F_{0}=f \circ H
$$

ii)

$$
|h(z)| \leq|z|^{m-\varepsilon}
$$

$$
\left|h\left(z_{2}\right)-h\left(z_{1}\right)\right| \leq \delta\left|z_{2}-z_{1}\right| .
$$

In particular, the mapping which we construct satisfies all the estimates present in Shcherbakov's work, but can actually be constructed smoothly to obtain a $C^{\infty}$ diffeomorphism off of the origin, and not merely a homeomorphism.

However, if we require the mapping to be real-analytic away from the origin with real-analytic inverse, the situation changes drastically. In particular, we show an equivalent condition for the existence of holomorphic conjugacy between germs of a special form as follows. 
Theorem 1.2. Suppose that the germ $f$ has the special form

$$
f(z)=z+a_{2} z^{2}+O\left(z^{3}\right),
$$

i.e., suppose that $m=2$ in (1.1). Suppose there exists a homeomorphism $R$ defined in a small neighborhood of the origin which is real-analytic except at the origin with real-analytic inverse, conjugating $f$ with $F_{0}$. Then, there exists a biholomorphism in a full neighborhood of the origin conjugating $f$ with $F_{0}$.

Hence, the $C_{*}^{\infty}$ classification agrees with the topological one (where $C_{*}^{\infty}$ means what we expect), but the real-analytic classification differs considerably. It is worth noting that Theorem 1.1 has been proved, in the case $C^{1}$ and $m=2$, in the dissertation of Jérôme Rey [4. However, his methods differ considerably from the ones presented here. Also, with regards to Theorem 1.2 it is important that $f$ is equivalent to $F_{0}$. It is easy to see that this theorem will fail for arbitrary $\tilde{f}$, that is, it is easy to find $f$ and $\tilde{f}$ which are real-analytically equivalent but holomorphically inequivalent. For example, let $c \in \mathbf{C}-\mathbf{R}$ and consider

$$
f(z)=z+z^{2}+c z^{3}
$$

and let

$$
\tilde{f}(z)=z+z^{2}+\bar{c} z^{3} .
$$

Then, $f$ is equivalent to $\tilde{f}$ via the antiholomorphic map $z \rightarrow \bar{z}$, but the two functions cannot be holomorphically equivalent, via the formal theory, since $c \neq \bar{c}$. In light of this, it is interesting that with the hypotheses of Theorem 1.2, the existence of a real-analytic map does indeed guarantee a holomorphic map, and rules out the antiholomorphic case.

This paper is part of the author's thesis, written under the direction of Xianghong Gong at the University of Wisconsin-Madison. The author is indebted to Professor Gong for valuable discussions.

\section{THE $C^{\infty}$ CASE}

In this section, we prove Theorem 1.1. Let $f$ be a germ as in (1.1). We begin by assuming that $m=2$. This is not necessary, as the case for arbitrary $m$ does not depend on this base case. However, the reader may find that the proofs are more easily seen in this case. The general case will be proven, in a similar fashion, at the end of the section.

By applying the change of variable

$$
z \mapsto \frac{z}{a_{2}}
$$

we can assume that the germ $f$ has the form

$$
f(z)=z+z^{2}+O\left(z^{3}\right) .
$$

Let us assume that $f$ is defined in a disc centered at 0 of radius $r$. In order to construct the homeomorphism, it is convenient to work in a neighborhood of $\infty$. Consider the change of variable

$$
A(z)=-\frac{1}{z}
$$

Note that this change of variable converts our time-one map $F_{0}$ into

$$
\widetilde{F}_{0}(\eta)=\left(A \circ F_{0} \circ A^{-1}\right)(\eta)=\eta+1 .
$$


Letting $R=\frac{1}{r}$, we may then assume that our map

$$
\tilde{f}(\eta)=\left(A \circ f \circ A^{-1}\right)(\eta)
$$

is defined outside of a box about zero, that is, $\tilde{f}$ is defined in a neighborhood of the form

$$
\Omega=\mathbf{C}-(\{|\operatorname{Re} \eta| \leq R\} \cap\{|\operatorname{Im} \eta| \leq R\}),
$$

where $R \geq \frac{1}{r}$. Furthermore, $\tilde{f}$ has the form

$$
\tilde{f}(\eta)=\frac{-1}{f\left(-\frac{1}{\eta}\right)}=\eta+1+G^{+}(\eta)
$$

where

$$
\left|G^{+}(\eta)\right|<\frac{c}{|\eta|}, \quad\left|G^{+^{\prime}}(\eta)\right|<\frac{c}{|\eta|^{2}} .
$$

Similarly

$$
\tilde{f}^{-1}(\eta)=\eta-1+G^{-}(\eta)
$$

where

$$
\left|G^{-}(\eta)\right|=\frac{c}{|\eta|}, \quad\left|G^{-^{\prime}}(\eta)\right|=\frac{c}{|\eta|^{2}} .
$$

Let us define the following sets:

$$
\begin{aligned}
A_{1}=\{\eta: \operatorname{Re} \eta \leq-R\}, & & A_{2}=\{\eta: \operatorname{Re} \eta \geq R\}, \\
B_{1}=\{\eta: \operatorname{Im} \eta \leq-R\}, & & B_{2}=\{\eta: \operatorname{Im} \eta \geq R\}, \\
\omega_{1}=\{\eta:-(R+1) \leq \operatorname{Re} \eta \leq-R\}, & & \omega_{2}=\{\eta: R \leq \operatorname{Re} \eta \leq R+1\} .
\end{aligned}
$$

We denote

$$
\widetilde{H}=I d+\hat{h} .
$$

Our goal is clear: we wish to construct $\hat{h}$ on the set $\Omega$ so that

$$
\tilde{f} \circ \widetilde{H}=\widetilde{H} \circ(I d+1) .
$$

In order to accomplish this, we will construct our mapping in two parts. Note that (2.5) yields the equation

$$
\hat{h}(\eta+1)=\hat{h}(\eta)+G^{+}(\eta+\hat{h}(\eta)) .
$$

Equivalently, the equation

$$
\hat{h}(\eta-1)=\hat{h}(\eta)+G^{-}(\eta+\hat{h}(\eta))
$$

holds for $f^{-1}$ and hence (2.6) and (2.7) yield the two functional equations

$$
\begin{aligned}
& \hat{h}(\eta+n)=\hat{h}(\eta)+\sum_{j=0}^{n-1} G^{+}(\eta+j+\hat{h}(\eta+j)), \\
& \hat{h}(\eta-n)=\hat{h}(\eta)+\sum_{j=0}^{n-1} G^{-}(\eta-j+\hat{h}(\eta-j)) .
\end{aligned}
$$

To begin, let us choose a function $\chi_{1} \in C^{\infty}\left(\omega_{1}\right)$ with the following properties (let $\eta=\xi+i \zeta)$ :

i) $\chi_{1}(\eta)=\chi_{1}(\operatorname{Re} \eta)$;

ii) $0 \leq \chi_{1} \leq 1,0 \leq \frac{\partial \chi_{1}}{\partial \xi} \leq 3$; 
iii) $\chi_{1}(\eta)=0$ if $-(1+R) \leq \operatorname{Re} \eta \leq-\left(\frac{3}{4}+R\right)$;

iv) $\chi_{1}(\eta)=1$ if $-\left(\frac{1}{4}+R\right) \leq \operatorname{Re} \eta \leq-R$.

In the set $\omega_{1}$, we define

$$
\hat{h}_{1}(\eta)=\chi_{1}(\eta) G^{+}(\eta-1) .
$$

We have that $\hat{h}_{1}(\eta)=0$ if $\operatorname{Re} \eta=-(1+R)$, and thus it is clear that $\hat{h}_{1}$ satisfies the functional equation (2.6). Thus, we may use equations (2.8) and (2.9) to extend $\hat{h}_{1}$ to a function defined on the set $\Omega_{1}=A_{1} \cup B_{1} \cup B_{2}$, and this extension is $C^{\infty}$ on this set; to see this, we only need to check on lines of the form $\{\operatorname{Re} \eta=C\}$ where $C$ is a constant of the form $-R+n$ for $n \in \mathbf{Z}$. Furthermore, by applying an inductive argument, it suffices to prove that the function is smooth in a small $\Omega$-neighborhood of the two lines $\{\operatorname{Re} \eta=-R\}$ and $\{\operatorname{Re} \eta=-R-1\}$. It is easy to check one of these; note that if $\operatorname{Re} \eta=-R-\delta$ for $\delta$ sufficiently small, then $\hat{h}_{1}(\eta)=G^{+}(\eta-1)$. Also, (2.8) tells us that if $\operatorname{Re} \eta=-R+\delta$, then

$$
\hat{h}_{1}(\eta)=\hat{h}_{1}(\eta-1)+G^{+}\left(\eta-1+\hat{h}_{1}(\eta-1)\right),
$$

and (2.10) tells us that the function is smooth over this line. To check the line $\{\operatorname{Re} \eta=-R-1\}$, we note that, on the right side of this line, $\hat{h}_{1}(\eta)=0$, while on the left side, we have, by (2.9),

$$
\hat{h}_{1}(\eta)=\hat{h}_{1}(\eta+1)+G^{-}\left(\eta+1+\hat{h}_{1}(\eta+1)\right) .
$$

In order for this to be 0 , we must have

$$
\hat{h}_{1}(\eta+1)=-G^{-}\left(\eta+1+\hat{h}_{1}(\eta+1)\right),
$$

and by (2.10), this implies that

$$
G^{+}(\eta)=-G^{-}\left(\eta+1+G^{+}(\eta)\right) .
$$

But this follows immediately from the fact that $\tilde{f}^{-1} \circ \tilde{f}=I d$. Hence, $\hat{h}_{1}$ is smooth on the set $\Omega_{1}$. Thus, if we define

$$
\widetilde{H}_{1}(\eta)=\eta+\hat{h}_{1}(\eta)
$$

then it is clear that, on $\Omega_{1}, \widetilde{H}_{1}$ satisfies 2.5) (we note here that the mapping which Shcherbakov constructs in his paper fails to be $C^{\infty}$ in the region $\Omega_{1}$ ).

Similarly, we choose $\chi_{2} \in C^{\infty}$ as above:

i) $\chi_{2}(\eta)=\chi_{2}(\operatorname{Re} \eta)$;

ii) $0 \leq \chi_{2} \leq 1,-3 \leq \frac{\partial \chi_{2}}{\partial \xi} \leq 0$;

iii) $\chi_{2}(\eta)=0$ if $\frac{3}{4}+R \leq \operatorname{Re} \eta \leq 1+R$;

iv) $\chi_{2}(\eta)=1$ if $R \leq \operatorname{Re} \eta \leq \frac{1}{4}+R$.

Defining

$$
\hat{h}_{2}(\eta)=\chi_{2}(\eta) G^{-}(\eta+1),
$$

we see that this function can be extended into $\Omega_{2}=A_{2} \cup B_{1} \cup B_{2}$ via (2.8) and (2.9). Writing

$$
\widetilde{H}_{2}(\eta)=\eta+\hat{h}_{2}(\eta)
$$

we see also that $\widetilde{H}_{1}$ satisfies (2.5). However, we must show that both $\widetilde{H}_{1}$ and $\widetilde{H}_{2}$ are actually invertible functions for suitably large $R$. 
We now prove three estimates for $\hat{h}_{1}$ on $\Omega_{1}$ (analogous estimates will hold for $h_{2}$ on $\Omega_{2}$ ). In particular, we will show that, given any $\delta>0$, we can find an $R$ so that $\hat{h}_{i}$ is Lipschitz with constant $\delta$. Thus, for $0<\delta<1$, this will then show that $\widetilde{H}_{i}$ is one-to-one $(i=1,2)$. Note that all of the results given here require a suitably large $\Omega=\Omega(R)$; that is, we will freely increase the size of $R$. The statements and proofs of these estimates are so similar to Shcherbakov's [5] that their proofs are presented merely for the convenience of the reader.

Proposition 2.1. Define $F=\left\{\eta:\left|\arg \eta-\frac{\pi}{2}\right| \leq \frac{\pi}{3}\right\}$. Let $R$ be suitably large. Then, for $\eta \in \omega_{1} \cap B_{2}$ and any $n \in \mathbf{N}$ so that $\eta+n \in F$, we have

$$
\left|\hat{h}_{1}(\eta+n)\right|<9 c .
$$

Recall that $c$ is the number given in (2.3); $\left|G^{+}(\eta)\right| \leq \frac{c}{|\eta|}$.

Proof. We prove this via induction. For $n=0$, we actually have $\left|\hat{h}_{1}(\eta)\right|<c$; this follows immediately from (2.3) and (2.10). Thus, suppose that $n>0$ and that (2.12) holds for all $j$ with $0 \leq j \leq n-1$. We will show that it holds also for $j=n$. We note the following:

$$
n<2|\eta|, \quad|\eta+j|>\frac{|\eta|}{2} \quad(0 \leq j \leq n-1)
$$

(both of these statements are easily determined by basic geometry). Via the induction hypothesis, for $R$ sufficiently large we have

$$
\begin{aligned}
\left|\eta+j+\hat{h}_{1}(\eta+j)\right| & >|\eta+j|-\left|\hat{h}_{1}(\eta+j)\right| \\
& >|\eta+j|-9 c \\
& >\frac{|\eta+j|}{2} .
\end{aligned}
$$

Putting this together, we get

$$
\begin{aligned}
\left|\hat{h}_{1}(\eta+n)\right| & =\left|\hat{h}_{1}(\eta)+\sum_{j=0}^{n-1} G^{+}\left(\eta+j+\hat{h}_{1}(\eta+j)\right)\right| \\
& \leq c+\sum_{j=0}^{n-1} \frac{c}{\left.\mid \eta+j+\hat{h}_{1}(\eta+j)\right) \mid} \\
& \leq c+\frac{4 c n}{|\eta|} \\
& <c+8 c=9 c .
\end{aligned}
$$

It is clear that Proposition 2.1 is true for $n<0$ and for the region $-F$ by analogous reasoning. Furthermore, we note that the proof of Proposition 2.1]implies the following:

Corollary 2.2. Let $\beta>0$. Then, we can find $R$ and $\alpha>0$ so that, in the region

$$
F_{\alpha}=\left\{\eta \in \mathbf{C}:\left|\arg \eta-\frac{\pi}{2}\right|<\alpha\right\},
$$

we have for $\eta \in F_{\alpha}$ that $\hat{h}(\eta) \leq \beta c$.

Hence, for $\alpha$ small enough, we can make $\hat{h}$ as small as we desire on the angular region $F_{\alpha}$. 
Proposition 2.3. Let $0<\varepsilon<1$ be given. Suppose also that $\eta \in F \cup(-F) \cup \omega_{1}$ and that $\eta+1 \in \Omega$, but $\eta+1 \notin F \cup(-F)$. Then, for $R$ large enough (depending only on $\varepsilon)$, we have

$$
\left|\hat{h}_{1}(\eta+n)\right|<|\eta+n|^{\varepsilon} .
$$

Proposition 2.3 tells us that the image of the set $\Omega$ under the map $\widetilde{H}$ will include the complement of a large disc in the plane.

Proof. Again, we use induction. For $n=0$ and $R$ sufficiently large, this follows from Proposition 2.1. Suppose then that $n>0$ (and hence that $\operatorname{Re} \eta>0$ ), and suppose that (2.13) holds for all $j$ with $0 \leq j \leq n$. Write $\eta^{\prime}=\eta+n$. Since $\eta^{\prime}+1 \notin F$ and $\operatorname{Re} \eta^{\prime}>0$, we have that $\left|\eta^{\prime}+1\right|>\left|\eta^{\prime}\right|+\frac{1}{2}$ (the key here is that $\left.\operatorname{Re}\left(\eta^{\prime}\right) \geq \sqrt{3} \operatorname{Im}\left(\eta^{\prime}\right)-1\right)$. This yields

$$
\left|\eta^{\prime}+1\right|^{\varepsilon}-\left|\hat{h}_{1}\left(\eta^{\prime}+1\right)\right|>\left|\eta^{\prime}+1\right|^{\varepsilon}-\left|\hat{h}_{1}\left(\eta^{\prime}\right)\right|-\left|G^{+}\left(\eta^{\prime}+\hat{h}_{1}\left(\eta^{\prime}\right)\right)\right| .
$$

Applying the induction hypothesis, we see that

$$
\left|\hat{h}_{1}\left(\eta^{\prime}\right)\right|<\left|\eta^{\prime}\right|^{\varepsilon}
$$

and

$$
\left|\eta^{\prime}+\hat{h}_{1}\left(\eta^{\prime}\right)\right|>\frac{\left|\eta^{\prime}\right|}{2}
$$

Therefore

$$
\begin{aligned}
\left|\eta^{\prime}+1\right|^{\varepsilon}-\left|\hat{h}_{1}\left(\eta^{\prime}+1\right)\right| & >\left(\left|\eta^{\prime}\right|+\frac{1}{2}\right)^{\varepsilon}-\left|\eta^{\prime}\right|^{\varepsilon}-\frac{2 c}{\left|\eta^{\prime}\right|} \\
& >\frac{\varepsilon}{2}\left|\eta^{\prime}\right|^{\varepsilon-1}-\left(\frac{\varepsilon(1-\varepsilon)}{8}|\eta|^{\varepsilon-2}+\frac{2 c}{\left|\eta^{\prime}\right|}\right) \\
& >0,
\end{aligned}
$$

again, if $R$ is chosen large enough.

Proposition 2.4. Suppose that $\eta \in K \subset \omega_{1}$, where $K$ is a disc of radius $\frac{1}{2}$, and suppose that $K+n \subset \Omega_{1}$, where $n \in \mathbf{Z}$. Then $\hat{h}_{1}$ satisfies a Lipschitz condition in the disc $K+n$ with Lipschitz constant $\delta=\delta(R)$, where $\delta(R) \rightarrow 0$ as $R \rightarrow \infty$.

Proof. We use induction. For $n=0$ we have

$$
\begin{aligned}
& \left|\hat{h}_{1}\left(\eta_{2}\right)-\hat{h}_{1}\left(\eta_{1}\right)\right| \\
& =\left|\chi_{1}\left(\eta_{2}\right) G^{+}\left(\eta_{2}-1\right)-\chi_{1}\left(\eta_{1}\right) G^{+}\left(\eta_{1}-1\right)\right| \\
& \leq\left|\chi_{1}\left(\eta_{2}\right)\right|\left|G^{+}\left(\eta_{2}-1\right)-G^{+}\left(\eta_{1}-1\right)\right|+\left|G^{+}\left(\eta_{1}-1\right)\right|\left|\chi_{1}\left(\eta_{2}\right)-\chi_{1}\left(\eta_{1}\right)\right| \\
& \leq\left|G^{+}\left(\eta_{2}-1\right)-G^{+}\left(\eta_{1}-1\right)\right|+3\left|G^{+}\left(\eta_{1}-1\right)\right|\left|\eta_{2}-\eta_{1}\right| \\
& \leq\left(\frac{c}{R^{2}}+\frac{3 c}{R}\right)\left|\eta_{2}-\eta_{1}\right| .
\end{aligned}
$$

Suppose now that $n>0$. Furthermore, assume by the induction hypothesis that $\hat{h}_{1}$ satisfies a Lipschitz condition in $K+n$ with constant $\delta_{n}<1$. Write $\eta_{j}^{\prime}=\eta_{j}+n$, 
$j=1,2$. We have

$$
\begin{aligned}
\left|\hat{h}_{1}\left(\eta_{2}^{\prime}+1\right)-\hat{h}_{1}\left(\eta_{1}^{\prime}+1\right)\right| & <\delta_{n}\left|\eta_{2}^{\prime}-\eta_{1}^{\prime}\right|+\frac{2 c}{\left|\eta_{1}^{\prime}\right|^{2}}\left|\eta_{2}^{\prime}-\eta_{1}^{\prime}+\hat{h}_{1}\left(\eta_{2}^{\prime}\right)-\hat{h}_{1}\left(\eta_{1}^{\prime}\right)\right| \\
& <\left|\eta_{2}^{\prime}-\eta_{1}^{\prime}\right|\left(\delta_{n}+\frac{2 c\left(1+\delta_{n}\right)}{\left|\eta_{1}^{\prime}\right|^{2}}\right) .
\end{aligned}
$$

We note the following fact:

$$
\left|\eta_{1}+n\right|<\frac{R+n}{4}
$$

(this follows via elementary geometry). Thus

$$
\delta_{n+1}<\delta_{n}+\frac{64 c}{|R+n|^{2}},
$$

and to complete the proof, we choose $R$ large enough so that

$$
64\left(\sum_{n=1}^{\infty}|R+n|^{-2}\right)+\frac{c}{R^{2}}+\frac{3 c}{R}<1 .
$$

As mentioned above, Proposition 2.4 guarantees that the mappings $\widetilde{H}_{j}, j=1,2$, are one-to-one in the domains

$$
\Omega_{j}=A_{j} \cup B_{1} \cup B_{2},
$$

provided that $R$ is large enough. Let us define regions of the form

$$
\widetilde{S}_{j}=\left\{\eta \in \Omega_{j}:|\arg \eta-\pi j|<\frac{\pi}{2}+\alpha\right\},
$$

where $0<\alpha<\frac{\pi}{3}$. If we write $\widetilde{H}_{j}^{-1}=I d+\tilde{h}_{j}$, Proposition 2.4 also guarantees that $\tilde{h}_{j}$ will satisfy a Lipschitz condition with constant $\delta^{\prime}=\frac{\delta}{1-\delta}$. Finally, Propositions 2.1 and 2.3 yield that the maps $\widetilde{H}_{j}$ will have domains and ranges containing and contained in sets of the form (2.15), again if $R$ is large enough.

To summarize, we have found two mappings

$$
\tilde{H}_{j}=I d+\hat{h}_{j}
$$

in the regions $\Omega_{j}$ so that

$$
\tilde{f} \circ \widetilde{H}_{j}=\widetilde{H}_{j} \circ(I d+1) .
$$

We now will patch these maps together to form a global map $\widetilde{H}$ in a neighborhood of $\infty$ which satisfies (2.16). It is here that our argument diverges most sharply from Shcherbakov's. In particular, in [5], Shcherbakov constructs a single mapping defined on the region $\Omega_{1}$ satisfying Propositions 2.1, 2.3, and 2.4. In order to then construct a mapping defined in a full neighborhood of $\infty$ satisfying (2.16), he presents a linear interpolation argument. Unfortunately, that argument will not produce a $C^{\infty}$ mapping. In order to counter this problem, we construct the two mappings $h_{1}$ and $h_{2}$. We then use a well-chosen partition of unity to patch these mappings together in a smooth way in a neighborhood of $\infty$; this is the argument presented now. First, choose $\alpha>0$ and $R$ so that we have $\beta<\frac{1}{1000000 c}$ and $\delta<\frac{1}{1000000}$. Let us define the regions $\widetilde{S}_{1}$ and $\widetilde{S}_{2}$ as in (2.15), taking $\alpha$ as chosen above. We define a mapping

$$
\Phi_{1}(\eta)=\widetilde{H}_{2}^{-1} \circ \widetilde{H}_{1}
$$


on the set

$$
\widetilde{S}_{1} \cap \widetilde{S}_{2} \cap\{\operatorname{Im} \eta>D>0\} .
$$

Note that, for $D$ large enough, the corollary to Proposition 2.1 insures that (2.17) is well defined. We note the following important property of $\Phi_{1}$ :

$$
\Phi_{1}(\eta+1)=\Phi_{1}(\eta)+1 .
$$

Hence, we may write

$$
\Phi_{1}(\eta)=\eta+p(\eta),
$$

where $p$ is smooth and 1-periodic. Note also that $p$ satisfies a Lipschitz condition with constant $\delta^{\prime \prime}=\frac{2 \delta}{1-\delta}$; this follows from Proposition 2.4. Hence, if we extend the mapping $p$ to a mapping $\tilde{p}$ which is defined in an upper half plane, then the mapping

$$
\widetilde{\Phi}_{1}(\eta)=\eta+\tilde{p}(\eta)
$$

with be one-to-one as well. Similarly, we can define a mapping on the set

$$
\widetilde{S}_{1} \cap \widetilde{S}_{2} \cap\{\operatorname{Im} \eta<-D<0\}
$$

given by

$$
\Phi_{2}=\widetilde{H}_{1}^{-1} \circ \widetilde{H}_{2}
$$

Writing

$$
\Phi_{2}(\eta)=\eta+q(\eta)
$$

we may subsequently extend $q$ to a mapping $\tilde{q}$ defined on a lower half plane, still satisfying a Lipschitz condition, and hence the mapping

$$
\widetilde{\Phi}_{2}(\eta)=\eta+\tilde{q}(\eta)
$$

will also be one-to-one. Now, we patch our maps. Choose a map $\psi_{1} \in C^{\infty}(\mathbf{C})$ with the following properties (again, let $\eta=\xi+i \zeta$ ):

i) $\psi_{1}(\eta)=\psi_{1}(\operatorname{Im} \eta)$

ii) $0 \leq \psi_{1} \leq 1,0 \leq \frac{\partial \psi}{\partial \zeta} \leq 3$,

iii) $\psi_{1}(\eta)=0$ if $\operatorname{Im} \eta \leq D$,

iv) $\psi_{1}(\eta)=1$ if $\operatorname{Im} \eta \geq D+1$.

Define the function

$$
\begin{aligned}
K(\eta) & =\left(1-\psi_{1}(\eta)\right) \eta+\psi_{1}(\eta)(\eta+\tilde{p}(\eta)) \\
& =\eta+\psi_{1}(\eta) \tilde{p}(\eta) .
\end{aligned}
$$

Note that $K$ is smooth on $\mathbf{C}$ (with the obvious adjustments), one-to-one, and that $K$ commutes with the unit shift. In similar fashion, choose $\psi_{2} \in C^{\infty}(\mathbf{C})$ so that

i) $\psi_{2}(\eta)=\psi_{2}(\operatorname{Im} \eta)$,

ii) $0 \leq \psi_{2} \leq 1,-3 \leq \frac{\partial \psi}{\partial \zeta} \leq 0$,

iii) $\psi_{2}(\eta)=0$ if $\operatorname{Im} \eta \geq-D$,

iv) $\psi_{2}(\eta)=1$ if $\operatorname{Im} \eta \leq-D-1$, 
and define

$$
\begin{aligned}
L(\eta) & =\left(1-\psi_{2}(\eta)\right) \eta+\psi_{2}(\eta)(\eta+\tilde{q}(\eta)) \\
& =\eta+\psi_{2}(\eta) \tilde{q}(\eta) .
\end{aligned}
$$

As with $K$, we see that $L$ is smooth on $\mathbf{C}$, one-to-one, and of course $L$ commutes with the unit shift. We use these two maps to patch together $\widetilde{H}_{1}$ and $\widetilde{H}_{2}$ :

$$
\widetilde{H}(\eta)= \begin{cases}\widetilde{H}_{1}(\eta) & \text { if } \operatorname{Re} \eta<-D, \operatorname{Im} \eta>0, \\ \left(\widetilde{H}_{2} \circ K\right)(\eta) & \text { if } 0 \leq \arg \eta \leq \frac{\pi}{2}+\gamma,|\eta|>D, \\ \widetilde{H}_{2}(\eta) & \text { if } \operatorname{Re} \eta>D, \operatorname{Im} \eta<0, \\ \left(\widetilde{H}_{1} \circ L\right)(\eta) & \text { if } \pi \leq \arg \eta \leq \frac{3 \pi}{2}+\gamma,|\eta|>D\end{cases}
$$

(here, $\gamma<\frac{\pi}{C}$ for $C$ very large). It is worth noting that the map $\widetilde{H}$ is well defined. To check this, note that if $\operatorname{Im} \eta=0$, then both $K=I d$ and $L=I d$. On the other hand, if $\operatorname{Re} \eta<-D$ and $\arg \eta<\frac{\pi}{2}+\gamma$, then $K=\widetilde{H}_{2}^{-1} \circ \widetilde{H}_{1}$, while if $\operatorname{Re} \eta>D$ and $\arg \eta<\frac{3 \pi}{2}+\gamma$, then $L=\widetilde{H}_{1}^{-1} \circ \widetilde{H}_{2}$, and hence the function agrees in the overlaps of the domains. Also, $\widetilde{H}$ is smooth in a neighborhood of $\infty$ (but NOT at $\infty$ !); this can be checked easily from the definitions (2.26) and (2.27). Finally, the mapping $\widetilde{H}$ does indeed conjugate $\tilde{f}$ with the unit shift. We check only one case, as the others follow analogously (or trivially):

$$
\begin{aligned}
\left(\widetilde{H}_{1} \circ K\right)^{-1} \circ \tilde{f} \circ\left(\widetilde{H}_{1} \circ K\right) & =K^{-1} \circ \widetilde{H}_{1}^{-1} \circ \tilde{f} \circ \widetilde{H}_{1} \circ K \\
& =K^{-1} \circ(I d+1) \circ K \\
& =K^{-1} \circ K \circ(I d+1) \\
& =I d+1 .
\end{aligned}
$$

If we write $\widetilde{H}(\eta)=\eta+\hat{h}(\eta)$, then Proposition 2.4 together with (2.26) and (2.27) guarantees that $\hat{h}$ satisfies a Lipschitz condition with constant $\delta$ as small as we like; taking $\delta<1$ insures that $\widetilde{H}$ possesses an inverse globally. Thus, we have constructed the mapping with the required properties in a neighborhood of $\infty$, and thus the mapping

$$
H(z)=-\frac{1}{\widetilde{H}\left(-\frac{1}{z}\right)}=\frac{z}{1-z \hat{h}\left(-\frac{1}{z}\right)}
$$

clearly satisfies (1.5) and (1.6), and (1.7) may be checked easily by using the righthand side of (2.29). Thus, the proof is complete for the case $m=2$.

Now, let us suppose that $m$ is arbitrary. Though the proof given here is very similar to the case $m=2$, it is worthwhile to look at the cases separately. First, by the formal classification theorem (e.g. see [2]), we can apply a (holomorphic) polynomial change of coordinate $p$ with degree no more than $m-1$ so that

$$
\left(p^{-1} \circ f \circ p\right)(z)=z+z^{m}+\mu z^{2 m-1}+O\left(z^{2 m}\right),
$$

and hence we assume that $f$ has this form. The change of variable which conjugates $g_{V}^{1}$ with the unit shift in a neighborhood of $\infty$ now becomes

$$
A_{m}(z)=-\frac{1}{(m-1) z^{m-1}},
$$


which unfortunately has a multi-valued inverse

$$
A_{m}^{-1}(z)=\sqrt[m-1]{\frac{-1}{(m-1) z}} .
$$

To remedy the situation, we look at the Riemann surface for the map $\sqrt[m-1]{z}$; let $0<r \ll 1$ and consider sectors of the form

$$
S_{j}=\left\{z \in \mathbf{C}:|z|<r,\left|\arg z-\frac{\pi j}{m-1}\right|<\alpha\right\},
$$

where $\frac{\pi}{2(m-1)}<\alpha<\frac{\pi}{m-1}, j=1, \ldots, 2(m-1)$. Note that these sectors cover a disc of radius $r$ with center at the origin. Let us look at the image of these sectors under the map (2.31) in the plane. When $j$ is odd, $S_{j}$ is mapped to

$$
\widetilde{S}_{j}=\left\{\eta \in \mathbf{C}:|\eta|>R,|\arg \eta-\pi|<\frac{\pi}{2}+\tilde{\alpha}\right\}
$$

while for $j$ even, $S_{j}$ is mapped to

$$
\widetilde{S}_{j}=\left\{\eta \in \mathbf{C}:|\eta|>R,|\arg \eta|<\frac{\pi}{2}+\tilde{\alpha}\right\},
$$

where $0<\tilde{\alpha}<\frac{\pi}{2}$. It is important to keep in mind that, for any given $j$, the sector $\widetilde{S}_{j}$ will only touch those sectors $\widetilde{S}_{j-1}$ and $\widetilde{S}_{j+1}$ (the exceptions being $\widetilde{S}_{1}$ and $\widetilde{S}_{2(m-1)}$, which overlap). We may think of these sectors as living in copies of $\mathbf{C}$, that is,

$$
\widetilde{S}_{j} \subseteq \mathbf{C} \times\{j\} .
$$

Given $\eta \in \widetilde{S}_{j}$, we say that $\eta \in \widetilde{S}_{j} \cap \widetilde{S}_{k}$ if we can find $\tilde{\eta} \in S_{k}$ so that

$$
A^{-1}(\eta)=A^{-1}(\tilde{\eta}) \text {. }
$$

It is easy to see that $\widetilde{S}_{1} \cap \widetilde{S}_{2}$ lies in an upper half plane, $\widetilde{S}_{2} \cap \widetilde{S}_{3}$ lies in a lower half plane, etc., until we reach $\widetilde{S}_{2(m-1)} \cap \widetilde{S}_{1}$, which lies in a lower half plane. This takes care of the multi-valuedness of $A^{-1}$.

We note that we can write

$$
\begin{aligned}
\tilde{f}(\eta) & =\left(A_{m} \circ f \circ A_{m}^{-1}\right)(\eta) \\
& =\frac{\eta}{\left(1-\frac{1}{(m-1) \eta}+\frac{1}{(m-1)^{2} \eta^{2}}+\cdots\right)^{m-1}} .
\end{aligned}
$$

Computing the Taylor series for the function $\left(\frac{1}{1-z}\right)^{m-1}$, we see that we can write

$$
\tilde{f}(\eta)=\eta+1+G^{+}(\eta)
$$

and similarly

$$
\tilde{f}^{-1}(\eta)=\eta-1+G^{-}(\eta)
$$

where $G^{+}$and $G^{-}$satisfy the estimates $(2.2)$. We are now basically in the situation of the case $m=2$; by following the constructions given in that case, we may construct maps $\widetilde{H}_{j}$ defined in the sectors $\widetilde{S}_{j}$ (for $j=1,2, \cdots, 2 m-2$ ) satisfying Propositions 2.1, 2.3 and 2.4 and of course satisfying

$$
\tilde{f} \circ \widetilde{H}_{j}=\widetilde{H}_{j} \circ(I d+1) .
$$

We now may look at the transition functions

$$
\Phi_{j}=\widetilde{H}_{j+1}^{-1} \circ \widetilde{H}_{j}
$$


for $j=1,2, \cdots, 2 m-3$ and

$$
\Phi_{2 m-2}=\widetilde{H}_{1}^{-1} \circ \widetilde{H}_{2 m-2} .
$$

As in the case $m=2$, these functions may be written as

$$
\Phi_{j}(\eta)=\eta+p_{j}(\eta)
$$

where $p_{j}$ is smooth and 1-periodic, for all $j=1, \cdots, 2 m-2$. We may then extend this mapping to $\tilde{p}_{j}$, defined in an upper half plane $\{\operatorname{Im} \eta>D\}$ in $\mathbf{C} \times\{j\}$ for $j$ odd, and a lower half plane $\{\operatorname{Im} \eta<-D\}$ in $\mathbf{C} \times\{j\}$ for $j$ even. Furthermore, the functions

$$
\widetilde{\Phi}_{j}(\eta)=\eta+\tilde{p}_{j}(\eta)
$$

will be one-to-one in these upper or lower half planes. Now, choose functions $\psi_{j} \in C^{\infty}(\mathbf{C})$ which satisfy

i) $\psi_{j}(\eta)=\psi_{j}(\operatorname{Im} \eta)$

ii) $0 \leq \psi_{j} \leq 1,0 \leq \frac{\partial \psi_{j}}{\partial(\operatorname{Im} \eta)} \leq 3$,

iii) $\psi_{2 n-1}(\eta)=0$ if $\operatorname{Im} \eta \leq D$ and $\psi_{2 n-1}(\eta)=1$ if $\operatorname{Im} \eta \geq D+1$,

iv) $\psi_{2 n}(\eta)=0$ if $\operatorname{Im} \eta \geq-D$ and $\psi_{2 n}(\eta)=1$ if $\operatorname{Im} \eta \leq-(D+1)$

(where $n=1,2, \cdots, m-1$ ). Using these maps, we can define smooth maps $K_{j}$, defined on $\mathbf{C} \times\{j\}$ as follows:

$$
\begin{aligned}
K_{j}(\eta) & =\left(1-\psi_{j}(\eta)\right) \eta+\psi_{j}(\eta)\left(\eta+\tilde{p}_{j}(\eta)\right) \\
& =\eta+\psi_{j}(\eta) \tilde{p}_{j}(\eta) .
\end{aligned}
$$

Note that $K_{j}$ is one-to-one on $\mathbf{C} \times\{j\}$ and commutes with the unit shift for all $j=1, \cdots, 2(m-1)$.

Finally, we can patch our mappings $\widetilde{H}_{j}$ together to form

$$
\widetilde{H}(\eta)= \begin{cases}\left(\widetilde{H}_{2 n-1}\right)(\eta) & \text { if } \eta \in \widetilde{S}_{2 n-1}, 0 \leq \arg \eta \leq \frac{\pi}{2}+\gamma, \\ \left(\widetilde{H}_{2 n} \circ K_{2 n-1}\right)(\eta) & \text { if } \eta \in \widetilde{S}_{2 n}, \operatorname{Re} \eta<-D, \operatorname{Im} \eta>0, \\ \left(\widetilde{H}_{2 n}\right)(\eta) & \text { if } \eta \in \widetilde{S}_{2 n}, \pi \leq \arg \eta \leq \frac{3 \pi}{2}+\gamma, \\ \left(\widetilde{H}_{2 n+1} \circ K_{2 n}\right)(\eta) & \text { if } \eta \in \widetilde{S}_{2 n+1}, \operatorname{Re} \eta>D, \operatorname{Im} \eta<0,\end{cases}
$$

where $n=1, \cdots, m-1$ (here, we define $\widetilde{H}_{2 m-1}=\widetilde{H}_{1}$ ). As in the case $m=2$, we choose $\gamma$ so that the mapping is well defined. This mapping satisfies a Lipschitz condition with constant as small as we like (via estimates analogous to Proposition 2.4). Now, we take this back into a neighborhood of the origin. We thus consider the mapping

$$
\begin{aligned}
H(z) & =\left(A_{m}^{-1} \circ \widetilde{H} \circ A_{m}\right)(z) \\
& =\sqrt[m-1]{\frac{-1}{(m-1)\left(\widetilde{H}\left(\frac{-1}{(m-1) z^{m-1}}\right)\right)}} \\
& =\sqrt[m-1]{\frac{-1}{(m-1)\left(\frac{-1}{(m-1) z^{m-1}}+\hat{h}\left(\frac{-1}{(m-1) z^{m-1}}\right)\right)}} \\
& =z\left(\sqrt[m-1]{\frac{1}{1-(m-1) z^{m-1} \hat{h}\left(\frac{-1}{(m-1) z^{m-1}}\right)}}\right) .
\end{aligned}
$$


Though this formula is quite ugly, by computing the Taylor series for the function $\sqrt[m-1]{\frac{1}{1-z}}$, we can rewrite the final line of $(2.42)$ as

$$
H(z)=z+z^{m} \hat{h}\left(\frac{-1}{(m-1) z^{m-1}}\right)+o\left(z^{m-\varepsilon}\right) .
$$

Now, it is clear that if we write $H(z)=z+h(z)$, then $h$ satisfies (1.6), and (1.7) may be checked in a fashion similar to the case $m=2$. Hence, the theorem is proved for arbitrary $m$.

\section{The REAL-ANALYTIC CASE}

In the previous section, we showed that given any two germs of the form

$$
f(z)=z+a_{2} z^{2}+\ldots
$$

where $a \neq 0$, these two germs were equivalent via a local homeomorphism $H$ which is a diffeomorphism off of the origin. In this section, we prove Theorem 1.2, which says that given $F(z)=\frac{z}{1-z}$, any local homeomorphism $R$ which is real-analytic away from the origin, with real-analytic inverse, conjugating a germ $f$ of the form (3.1) with the germ $F$ must necessarily give rise to a biholomorphic map $H$ in a full neighborhood of the origin conjugating $f$ with $F$. In particular, by the formal theory, this will put restrictions on the formal invariant $a_{3} / a_{2}^{2}$ of the function $f$ (as mentioned in the introduction).

The key is the following "basic lemma" of Voronin [6]:

Lemma 3.1. Suppose that $f$ is a germ as in (3.1), defined in a small disc $D(0, \rho)$, and define the set

$$
\Omega_{\rho}=\left\{\operatorname{Re\eta }>\frac{1}{\rho}\right\} \cup\left\{|\operatorname{Im} \eta|>\frac{1}{\rho}\right\} .
$$

Suppose that $G$ is a quasi-conformal homeomorphism of $\Omega_{\rho}$ into $D$ so that

$$
G^{-1} \circ f \circ G=I d+1 .
$$

Define $G\left(\Omega_{\rho}\right)=\Omega$. Then, there exists an injective holomorphic mapping $H: \Omega \rightarrow$ C so that, if we define $\widetilde{\Omega}=H(\Omega)$, then

$$
H \circ f \circ H^{-1}=I d+1
$$

on $\widetilde{\Omega}$. The set $\widetilde{\Omega}$ will contain a set of the form $\Omega_{\varepsilon}$ for $\varepsilon>0$ sufficiently small. Moreover, the map $H$ is unique in the following sense: given another map $\widetilde{H}$ satisfying the conclusions above, there exists $c \in \mathbf{C}$ and $\varepsilon^{\prime}$ so that $\widetilde{H}=H+c$ on $\Omega_{\varepsilon^{\prime}} \subseteq \Omega$.

Recall that a homeomorphism $G: \Omega \rightarrow \mathbf{C}$ is called quasi-conformal if $G$ has locally integrable generalized derivatives satisfying the estimate

$$
\left|\frac{\partial G}{\partial \bar{z}}\right| \leq k\left|\frac{\partial G}{\partial z}\right|
$$

Another characterization of quasi-conformal mappings is the following: define the quantity

$$
K_{G}\left(z_{0}\right)=\varlimsup_{r \rightarrow 0}\left(\frac{\sup _{\left|z-z_{0}\right|=r}\left|f(z)-f\left(z_{0}\right)\right|}{\inf _{\left|z-z_{0}\right|=r}\left|f(z)-f\left(z_{0}\right)\right|}\right)
$$

this quantity is called the quasi-conformal deviation of $G$ at $z_{0}$. Then, the mapping $G$ is quasi-conformal if and only if $K_{G}\left(z_{0}\right)<\infty$ for all $z_{0} \in \Omega$ and $K=\left|K_{G}\right|_{L^{\infty}}<$ 
$\infty$. Using this condition, it is clear that the homeomorphism $H$ constructed in section 2 is quasi-conformal as long as the Lipschitz constant $\delta<1$, for then we have

$$
\left|K_{H}\left(z_{0}\right)\right| \leq \frac{1+\delta}{1-\delta} .
$$

Hence, we may assume that, given a holomorphic mapping $f$ defined on some disc $D(0, \rho)$, we have a local homeomorphism $R$ defined on $D$, which is real-analytic with real-analytic inverse away from 0 , so that

$$
R \circ f \circ R^{-1}(z)=\frac{z}{1-z} .
$$

Also, by Lemma 3.1 (the "basic lemma"), we can find a holomorphic map $H$, defined on some open subset $U$ of $D$, so that

$$
H \circ f \circ H^{-1}(\eta)=\eta+1,
$$

and satisfying that $\Omega_{\varepsilon} \subseteq H(U)$ for some $\varepsilon>0$ sufficiently small. Again, let $A(z)=-\frac{1}{z}$, so that we have

$$
A \circ R \circ f \circ R^{-1} \circ A^{-1}=I d+1 .
$$

Then, (3.6) and (3.7) yield that, on a set of the form $\Omega_{\varepsilon^{\prime}}, 0<\varepsilon^{\prime}<\varepsilon$ we have

$$
A \circ R \circ H^{-1}(\eta+1)=A \circ R \circ H^{-1}(\eta)+1,
$$

so that we may write

$$
A \circ R \circ H^{-1}(\eta)=\eta+p(\eta),
$$

where $p$ is 1-periodic and real-analytic on $\Omega_{\varepsilon^{\prime}}$. Extending $p$ to $\tilde{p}$ defined on the complex plane, we write

$$
B(\eta)=\eta+\tilde{p}(\eta) .
$$

It is clear that $B$ is injective; to see this, suppose that

$$
B\left(\eta_{2}\right)=B\left(\eta_{1}\right) .
$$

Choose a single $n \in \mathbf{Z}$ and $\eta_{i}^{\prime} \in \Omega_{\varepsilon^{\prime}}$, so that

$$
\eta_{i}=\eta_{i}^{\prime}+n,
$$

for $i=1,2$. Now, we have

$$
B\left(\eta_{1}^{\prime}+n\right)=B\left(\eta_{2}^{\prime}+n\right),
$$

which implies that $B\left(\eta_{1}^{\prime}\right)=B\left(\eta_{2}^{\prime}\right)$. Since $B$ is certainly injective in the region $\Omega_{\varepsilon^{\prime}}$, we must have $\eta_{1}^{\prime}=\eta_{2}^{\prime}$, which then shows that $\eta_{1}=\eta_{2}$.

It is important to note that

$$
|B(\eta)| \rightarrow \infty \text { as }|\eta| \rightarrow \infty .
$$

In order to prove (3.13), we need the following lemma:

Lemma 3.2. Suppose that $f$ is holomorphic on the disc $D(0, \delta)$, and let $H$ be the map given by the "basic lemma" (i.e. Lemma 3.1) with domain $\Omega$ (and recall that $H(\Omega) \supseteq \Omega_{\rho}$ for some $\left.\rho>0\right)$. Let $0<\delta^{\prime}<\delta$ be given. Then, we can find $\rho^{\prime}$ so that

$$
H\left(D\left(0, \delta^{\prime}\right) \cap \Omega\right) \supseteq \Omega_{\rho^{\prime}} .
$$


Proof. This follows from the uniqueness property of Lemma 3.1. To be more precise, the mapping $f$ is defined on the disc $D\left(0, \delta^{\prime}\right)$, and hence Lemma 3.1 tells us that we can find a map $\widetilde{H}$, defined on a domain $\widetilde{\Omega}$ with $\widetilde{H}(\widetilde{\Omega}) \supseteq \Omega_{\rho^{\prime \prime}}$, with $\rho^{\prime \prime}>0$, which conjugates $f$ with the unit shift. By uniqueness, on the set $\widetilde{\Omega}$, we can write

$$
H=\widetilde{H}+c
$$

where $c \in \mathbf{C}$. Thus, if we write $L_{c}(z)=z+c$, then $H(\widetilde{\Omega})$ contains the set $L_{c} \Omega_{\rho^{\prime \prime}} \supseteq$ $\Omega_{\rho^{\prime}}$, completing the proof of the lemma.

We now prove (3.13). Let $C>0$. By Lemma 3.2 we can choose $\varepsilon^{\prime}$ so that, on the set $\Omega_{\varepsilon^{\prime}}$, we have $|B|>C$. Now, consider the set

$$
\mathbf{C}-\Omega_{\varepsilon^{\prime}}=\left\{\eta \in \mathbf{C}: \operatorname{Re} \eta \leq \frac{1}{\varepsilon^{\prime}} \text { and }|\operatorname{Im} \eta| \leq \frac{1}{\varepsilon^{\prime}}\right\} .
$$

On this set, the values of $\tilde{p}$ are completely defined by its values in a closed rectangle of base 1 and height $\frac{2}{\varepsilon^{\prime}}$; in particular, $\tilde{p}$ is bounded on the set $\mathbf{C}-\Omega_{\varepsilon^{\prime}}$. Thus, we can choose $\varepsilon^{\prime \prime}>0$ so that, if $\eta \in \mathbf{C}-\Omega_{\varepsilon^{\prime}}$ and $\operatorname{Re} \eta<-\varepsilon^{\prime \prime}$, then $|B|>C$. Thus, $|B|>C$ on a full neighborhood of $\infty$, and this proves (3.13).

Using (3.13), we see that the map $R^{-1} \circ A^{-1} \circ B$ is real-analytic in a neighborhood $U$ of $\infty$ and agrees with the map $H^{-1}$ on some open subset $U^{\prime} \subseteq U$. Thus, this map defines a real-analytic, and hence holomorphic, extension of $H^{-1}$ to $U$. Hence, the map $\widetilde{H}=R^{-1} \circ A^{-1} \circ B \circ A$ is defined and holomorphic in a punctured neighborhood of the origin, and is bounded in a full neighborhood of the origin, by (3.13). By the theorem on removable singularities, $\widetilde{H}$ may be extended to a full neighborhood of the origin, with $\widetilde{H}(0)=0$ (again, by (3.13) $)$. Furthermore, $\widetilde{H}$ has a holomorphic inverse by virtue of injectivity. Finally, it is clear that

$$
\left(\widetilde{H}^{-1} \circ f \circ \widetilde{H}\right)(z)=\frac{z}{1-z}
$$

(for example, one could apply the identity theorem), which completes the proof.

From here, a question comes to mind. As mentioned in the introduction, Theorem 1.2 is obviously false in general. Thus, we can ask the following:

Question 1. Given that $f$ and $\tilde{f}$ are conjugate via a mapping satisfying the hypotheses of Theorem 1.2, under what conditions on $\tilde{f}$ will the conclusion of Theorem 1.2 hold?

The proof of Theorem 1.2 given here depends largely on the fact that the holomorphic mapping which takes $F_{0}(z)=\frac{z}{1-z}$ to $\widetilde{F}_{0}(\eta)=\eta+1$ in a neighborhood of $\infty$ is globally defined in a punctured neighborhood of the origin; $A(z)=-\frac{1}{z}$. This is not the case in general. For example, consider the time-one map $g_{\mu}^{1}$ of the vector field $V(z)=\frac{z^{2}}{1+\mu z} \frac{\partial}{\partial z}$ for $\mu \neq 0$. Then, the map $A_{\mu}(z)=-\frac{1}{z}+\mu \log z$ will take $g_{\mu}^{1}$ to $F_{0}$, but cannot be globally defined in a punctured neighborhood of the origin. Also, the proof depends on the fact that $m=2$; if we instead assume that $f$ is conjugate to the time-one map of the vector field $V(z)=z^{m} \frac{\partial}{\partial z}$ for $m \geq 3$, this proof will fail. It would be nice to see precise conditions under which we have such a characterization of holomorphic conjugacy.

\section{REFERENCES}

1. Patrick Ahern and Jean-Pierre Rosay, Entire functions, in the classification of differentiable germs tangent to the identity, in one or two variables, Trans. Amer. Math. Soc., 310 (1995), 543-572. MR.1276933 (95d:30038) 
2. Y. S. Il'yashenko, Nonlinear Stokes Phenomena, Adv. in Soviet Math., vol. 14, Amer. Math. Soc., Providence, RI, 1993. MR,1206041 (94d:32031)

3. Jean Martinet and Jean-Pierre Ramis, Classification analytique des équations différentielles non linéaires résonnantes du premier ordre, Ann. Scient. École Norm. Sup., série 4, 16 (1983), 571-621. MR740592 (86k:34034)

4. Jérôme Rey, Difféomorphismes Résonnants de $(\mathbb{C}, 0)$, Thesis, L'Université Paul Sabatier de Toulouse (1996).

5. A. A. Shcherbakov, Topological classification of germs of conformal mappings with identical linear part, Vestnik Moskov. Univ. Ser. I Mat. Mekh., 1982, no. 3, 52-57; English transl. in Moscow Univ. Math. Bull. 37 (1982). MR671059 (84f:58014)

6. S. M. Voronin, Analytic classification of germs of conformal maps $(\mathbb{C}, 0) \rightarrow(\mathbb{C}, 0)$ with identical linear part, Funktsional. Anal. i Prilozhen. 15 (1981), no.1, 1-17; English transl. in Functional Anal. Appl. 15 (1981). MR609790 (82h:58008)

Department of Mathematics, University of Wisconsin, Madison, Wisconsin 53706

Current address: Department of Mathematics, Purdue University, West Lafayette, Indiana 47907

E-mail address: majenkin@math.purdue.edu 